Colloque C3, suppl. au Journal de Physique III, Vol. 1, octobre 1991

\title{
INFLUENCE OF LOADING PATHS ON THE MECHANICAL RESPONSE AND SUBSTRUCTURE EVOLUTION OF SHOCK-LOADED COPPER
}

\author{
G.T. GRAY III and C.E. MORRIS
}

Los Alamos National Laboratory, Los Alamos, New Mexico 87545, U.S.A.

Résumé: Des expériences de choc ont été réalisées sur des cibles de cuivre afin d'étudier l'influence de la vitesse de chargement et de l'amplitude de la contrainte sur l'accumulation des défauts et les propriétés mécaniques post-choc. Le temps de montée đu choc variait approximativement depuis une nanoseconde jusqu'à une microseconde dans le cas de chargements quasi-isentropiques. L'amplitude et la durée de la pression appliquée étaient identiques pour tous les essais. A $10 \mathrm{GPa}$ de pression maximum atteinte l'accumulation des défauts et la limite d'élasticité du cuivre post-choc sont les mêmes pour un chargement par choc simple et un charmgement par chocs multiples. L'effet de la vitesse de chargement sur la sousstructure post-choc et la réponse mécanique du cuivre impacté est supposée être directement relié à l'importance du déplacement des dislocations avant interaction avec d'autres dislocations et à l'importance du déplacement réversible des dislocations et leur annihilation résultante au cours de la phase de détente du cycle choc-détente.

\begin{abstract}
Shock recovery experiments on copper have been conducted to investigate the influence of loading rate and stress amplitude on defect storage and post-shock mechanical properties. The shock risetimes varied approximately from one nanosecond for the shock experiments to one microsecond for quasi-isentropic loading experiments. All the experiments had the same peak pressure and pulse duration. Attaining a peak pressure of $10 \mathrm{GPa}$ through multiple shocks or a single shock produced similar results for defect storage and post-shock yield strength. Decreasing the strain-rate of loading is shown to increase both the defect storage and post-shock yield strength of copper. The effect of loading rate on post-shock substructure and mechanical response of impacted copper is postulated to be directly related to the amount of dislocation motion before interaction with other dislocations and to the amount of reversible dislocation motion and resultant annihilation during the rarefaction portion of the shock-release cycle.
\end{abstract}

\section{1.- Introduction}

Shock loading in most metals and alloys produces greater hardening than quasi-static deformation to the same total strain, particularly if the metal undergoes a polymorphic phase transition, such as observed in iron $/ 1,2 /$. This phenomenon has been attributed to the very high strain rates associated with shock loading and the subsonic restriction on dislocation motion requiring the generation and storage of a larger dislocation density during the shock process than for quasi-static processes $/ 2 \%$. The increased defect storage and post-shock mechanical response of shock-loaded metals with increasing shock pressure has been linked to the increasing strain rate applied to the material. In the case of copper shock loaded to 10 GPa the high rates during the shock rise are related to the subnanosecond risetime of the shock pulse. This high loading rate leads to an imposed strain rate of the order of 106 to $10^{8} \mathrm{~s}^{-1}$. The highest strain-rate tests obtainable by non-shock experiments are near $10^{4} \mathrm{~s}^{-1}$ and can be done in the split-Hopkinson bar. Direct comparison of the deformation mechanisms in these two tests is complicated by factors such as: 1) the inherent stress-reversal loading path nature of shock recovery experiments, 2 ) the difference in the stress state between the uniaxial-strain shocks and uniaxial-stress split-Hopkinson bar tests, and 3) the two or more orders of magnitude difference in strain rate between the two tests. 
Layered impactors/3/ and the development of graded-density impactors 'pillows' /4,5/ and 'MIVAR' /6/ have allowed dynamic quasi-isentropic compression studies of a variety of materials. These techniques allow examination of material response in the gap between shock and conventional high-strain-rate tests Experimental results on $\mathrm{Al} / 4 / \mathrm{W} / 5 /$ and $\mathrm{Cu} / 3$ / have all determined that the quasi-isentrope lies above the shock Hugoniot suggesting that the strength of the materials might be increasing much more rapidly with pressure under quasi-isentropic loading than it does under shock loading. Due to the lower applied strain rates during quasi-isentropic loading, $\sim 10^{5} \mathrm{~s}^{-1}$, this result is somewhat counter intuitive given the normal extrapolations based on high-rate tests. One explanation for this phenomenon is a greatly reduced amount of heat evolution in quasi-isentropic loading compared to the shock. At high shock pressures this heating could be sufficient enough to drastically reduce the yield response of the material compared to auasiisentropic loading. This concept is however difficult to support at low pressures (<20 GPa) where shock heating is minimal, yet the quasi-isentrope still lies above the Hugoniot $/ 6 /$. In this paper the influence of loading rate and stress amplitude during shock loading on the defect generation and storage processes has been examined in OFE copper. Shock-recovery experimental techniques have been utilized to obtain samples that have been impacted to a $10 \mathrm{GPa}$ and $1 \mu$ s duration over a risetime range from $<1 \mathrm{~ns}$ to $1 \mu \mathrm{s}$.

\section{2.-Experimental}

The influence of loading path on the post-shock structure/property behavior of copper was investigated by impacting OFE copper with three different impactor configurations. These include: (1) 2.36-mm-thick copper impactor impacting a copper assembly at $518 \mathrm{~m} / \mathrm{s}$ to generate a $10 \mathrm{GPa}$ shock in a single step, (2) a multiple shock experiment with a composite plexiglas/copper impactor yielding a step-loading wave to 10 GPa peak pressure, and (3) a $\sim 1 \mu$ s ramp loading wave to $10 \mathrm{GPa}$ utilizing a composite "pillow" /3/ copper impactor. Characteristics-code calculations were utilized to design the experiments. The thickness of the copper portion of the symmetric or composite impactor was adjusted to maintain a constant $1 \mu \mathrm{sec}$ pulse duration for all three loading paths. Figure 1 shows a schematic illustration of the variation in loading-waves, and coincident variation in strain rates with the three different impactor configurations.

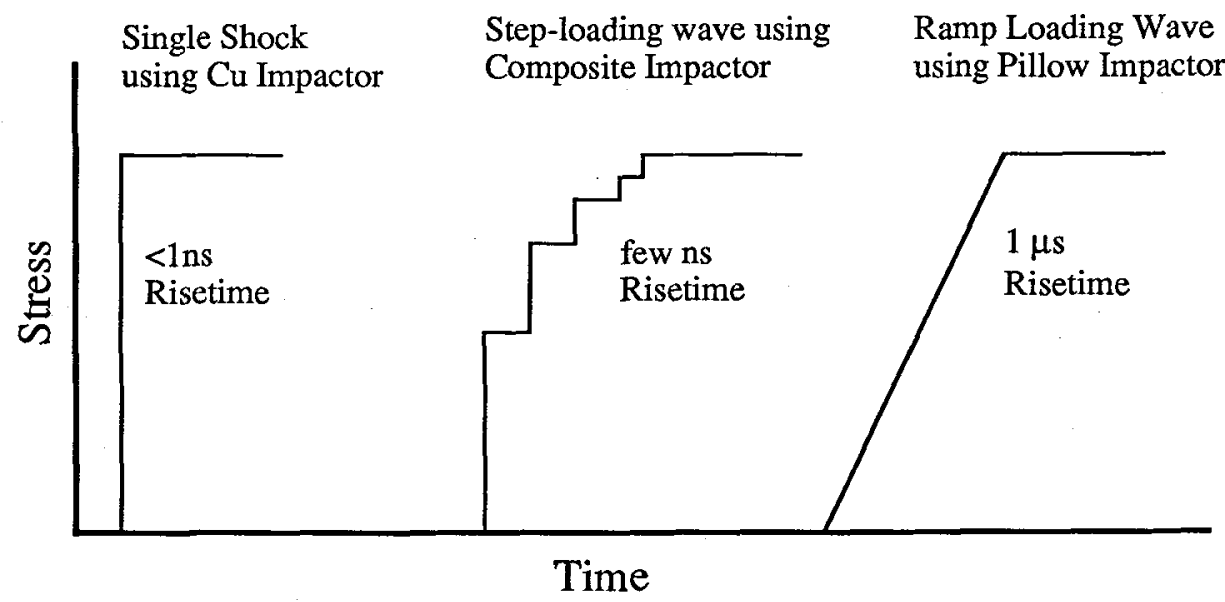

Fig. 1.- Schematic drawing of three different loading paths studied.

The motivation for studying these three different loading paths was to identify the various factors influencing dislocation motion in the "shock" process. The symmetric impact experiments have the largest stress gradients and shortest risetimes $(<1 \mathrm{~ns})$. For the step-loading experiments the peak pressure is attained through a series of small shocks. The risetime (few ns) of these smaller shocks is larger because of the decreased amplitude of the individual shocks, and also the stress gradients are smaller for the same reason. Comparison of these two experiments will illustrate the importance of the magnitude of the stress gradients in the shock front. The ramp-wave experiments were done to examine the role strain rate plays in the generation of dislocations. The strain rate in these experiments is three orders of magnitude less than in the other two types of experiments. Through the comparison of these experiments it is hoped a better understanding of the dislocation generation in the "shock" process can be attained. 
The samples were fabricated from oxygen-free-electronic (OFE) copper and annealed in vacuum at $600^{\circ} \mathrm{C}$ for 1 hour, which yielded an average grain diameter of $40 \mu \mathrm{m}$. Acceleration of the impactors was accomplished utilizing an 80-mm single-stage-gas launcher. The impactors were backed by a low impedance glass microballoon foam and all impacts were conducted in a vacuum (less than $2 \mathrm{~Pa}$ ). The specimen assembly consisted of a $5.08-\mathrm{mm}$-thick, 38-mm-dia sample, positioned behind a 38-mm-dia, 2.54-mm-thick cover plate. Both components were tightly fit into a bored recess of a 70 - tapered, 12.7mm-thick, central momentum disc. The sample was protected from spallation by backing the central momentum disc with a 3.8-mm-thick spall plate. The central disk and spall plate were further surrounded by two concentric momentum trapping rings with outside diameters of 69.8 and $82.5 \mathrm{~mm}$. The central momentum disc, outer momentum trapping rings, and spall plate were fabricated from phosphor bronze to help ensure "soft" recovery. Soft recovery and simultaneous cooling were achieved by decelerating the central momentum disc in a water catch chamber positioned immediately behind the impact area. Utilization of the techniques described yielded samples with a residual strain (defined here as the change in sample thickness divided by the starting sample thickness) of $<2 \%$. A detailed description of the importance of "soft" shock recovery techniques to post-shock structure / property behavior and detailed techniques utilized have been previously published $\Pi, 8 /$.

Compression specimens were electro-discharge machined (EDM) from the shock-loaded disk to allow postimpact measurement of the reload yield behavior. The compression axis was parallel to the shock direction. Samples for transmission electron microscopy (TEM) were sectioned from the shocked disk in a direction normal to the impact surface. The TEM samples were purposely not sectioned near the sample surfaces because of the known influences of contact stresses on the dislocation substructure directly adjoining the surface/8/. Discs 3-mm in diameter were punched and electropolished in a solution of $20 \%$ $\mathrm{HNO}_{3}$ and methanol at $-40^{\circ} \mathrm{C}$, using 12 volts in a Struer's Electropolisher. Observation of the foils was done using a JEOL $2000 \mathrm{EX}$ at $200 \mathrm{kV}$. Characterization of the deformation substructures was performed using a constant [110] zone axis to keep constant the number of visible dislocations contributing to the image.

\section{3.- Results and Discussion}

Figure 2 shows the reload compression true stress-true strain response of shock-loaded copper illustrating the effect of loading rate on the post-impact mechanical response. Ramp loading is seen to result in increased shock hardening (post-shock yield of $\sim 260 \mathrm{MPa}$ ) compared to symmetric loading (220 MPa) or the step-loading ( $\sim 200 \mathrm{MPa})$. The $\sim 20 \mathrm{MPa}$ yield difference between the symmetric-loading and steploading is considered beyond experimental error suggesting it may be real. Further experiments are required to quantify the differences between single shock and multiple shock-loading.

The deformation substructure in all three loading experiments consisted of dislocation cells. In the $10 \mathrm{GPa}$ symmetric-Cu case, the substructure was seen to consist of loosely-tangled dislocation cells, approximately $0.4 \mu \mathrm{m}$ in diameter, with numerous dislocation loops interspersed within the cell interiors and cell walls(Figure 3). This observation is similar with many substructural observations in the literature on cell formation in shock-loaded high-stacking fault energy (SFE) single-crystal and polycrystalline FCC metals 19\%. The formation of dislocation cells in shock-loaded copper is consistent with the relatively high SFE of copper, which aids cross-slip and thereby cell formation. The substructure of the step-loaded sample also consisted of dislocation cells, however, the cell walls were found to be more well-defined in appearance (Figure 4). Many individual dislocations and dislocation loops can also be resolved within the cell walls formed in the step-loaded sample. Finally, the ramp-loaded sample exhibits very well-defined cell walls with a high density of dislocation networks in the walls (Figure 5). In addition to the dense cell wall structure, isolated dislocation loops were still observed within the cell interiors. Both the reload mechanical and substructure response support the concept of an increased amount of dislocation storage resulting from ramp loading. Qualitatively, this post-shock behavior is consistent with the shock-wave data showing the quasi-isentrope lying above the Hugoniot.

The difference between the ramp loading and shock loading on post-shock substructure and mechanical response is believed to directly reflect the amount of dislocation storage in the shock front prior to the reversibility of plasticity during the shock release. Given the $10^{3}$ difference in risetime and approximately same total shock strain to be accommodated in both cases, the symmetric shock in copper will produce a considerably higher number of dislocations moving a short distance compared to fewer dislocations moving larger distances in the ramp case. In the conventional shock impact, dislocations moving at 0.1 the shear wave velocity in copper $(2330 \mathrm{~m} / \mathrm{s})$ could traverse $\sim 0.23 \mu \mathrm{m}$ during the $1 \mathrm{~ns}$ risetime. In the 
$1 \mu \mathrm{s}-$-rise for the ramp loading case, given the same dislocation velocity, the distance traveled is $\sim 230 \mu \mathrm{m}$. In a heavily deformed metal with a dislocation density $(\rho)$ of $10^{10} \mathrm{~cm}^{-2}$ the distance between dislocations is approximately $\rho^{-1 / 2}$ or in this case $0.1 \mu \mathrm{m}$. In the case of the shock, the approximate distance the dislocation can move is of the similar order of the dislocation spacing and considerably smaller than the grain size in most polycrystals. Conversely, in the ramp-loading impact, while the total number of dislocations required to achieve the applied strain-rate is much lower, the chance of dislocations intersecting either other dislocations or grain boundaries is significantly higher.

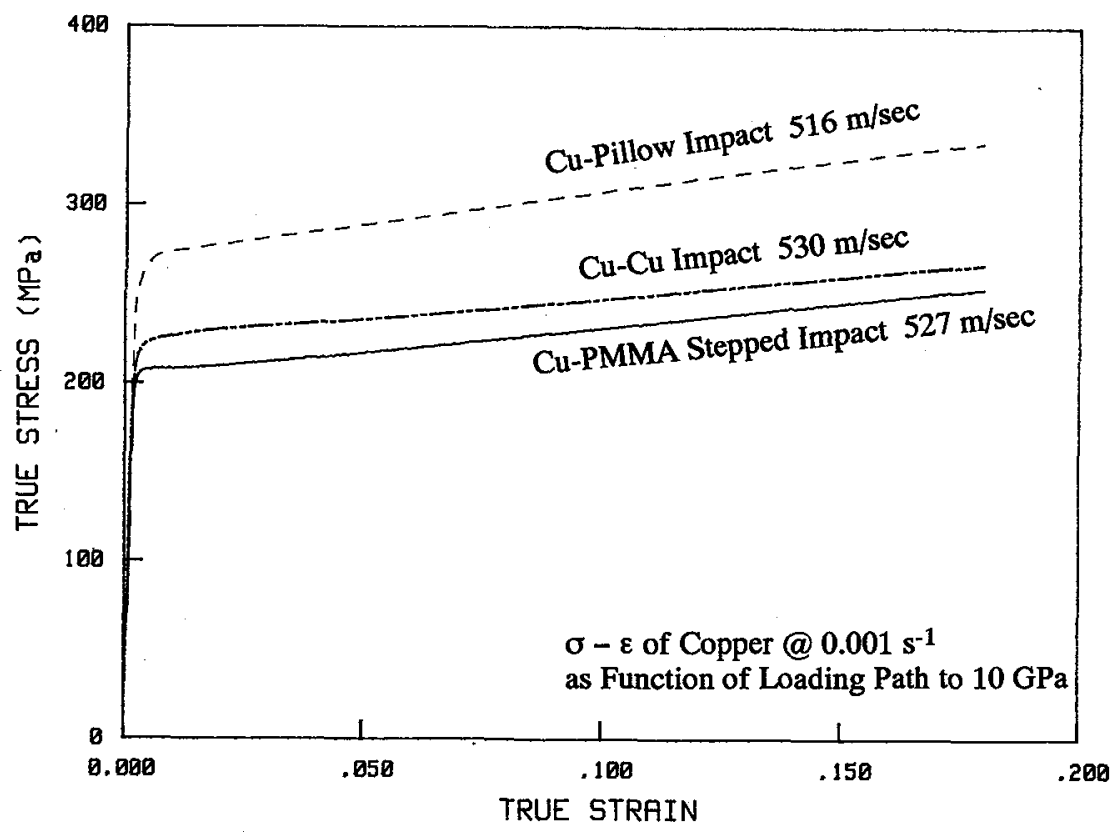

Fig. 2.- Stress-strain response of copper 'shock' loaded to $10 \mathrm{GPa}$ on three different loading paths.

The greatly increased dislocation-dislocation interactions are expected to lead to increased dislocation tangling and thereby increased strain hardening. In addition, in ramp loading, the increased time (i.e. lower strain rate), should also increase the total defect storage during the shock by facilitating more crossslip, which is a thermally-activated process. Increased out of slip-plane dislocation motion should greatly reduce the amount of easily reversible dislocation motion and annihilation during the release portion of the shock cycle. In the case of the shock, the short travel distances of a large number of dislocations will favor more reversibility and annihilation of dislocations at their sources. As postulated, this model would predict enhanced dislocation storage under quasi-isentropic loading compared to shock loading. This difference should be manifested after both the shock rise, in line with the wave-profile data, and following the release, consistent with the recovery findings in this study. The current experimental results also suggest that the influence of plastic flow in two distinct directions during the shock release process, influencing defect storage, which we first introduced in regards to pulse duration effects $/ 10 \%$. This strain reversibility may be influencing many more issues of shock behavior than we initially anticipated. Additional studies designed to probe a range of loading paths on a matrix of materials will be conducted to study the influence of loading paths on structure / property behavior. 


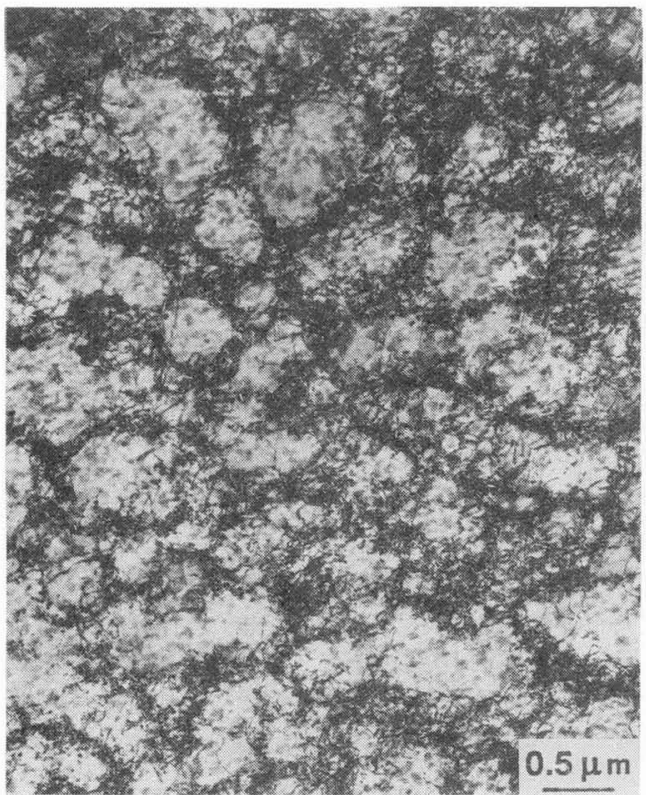

Fig. 3.- Loosely-tangled dislocation cells in symmetric shock loading experiments.

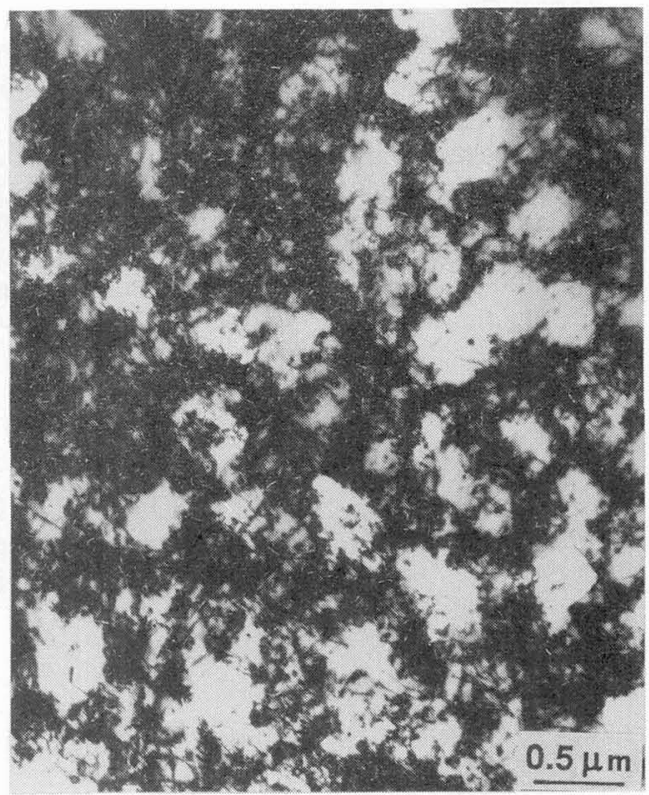

Fig. 4.- Dislocation cells formed in step-loading shock experiments.

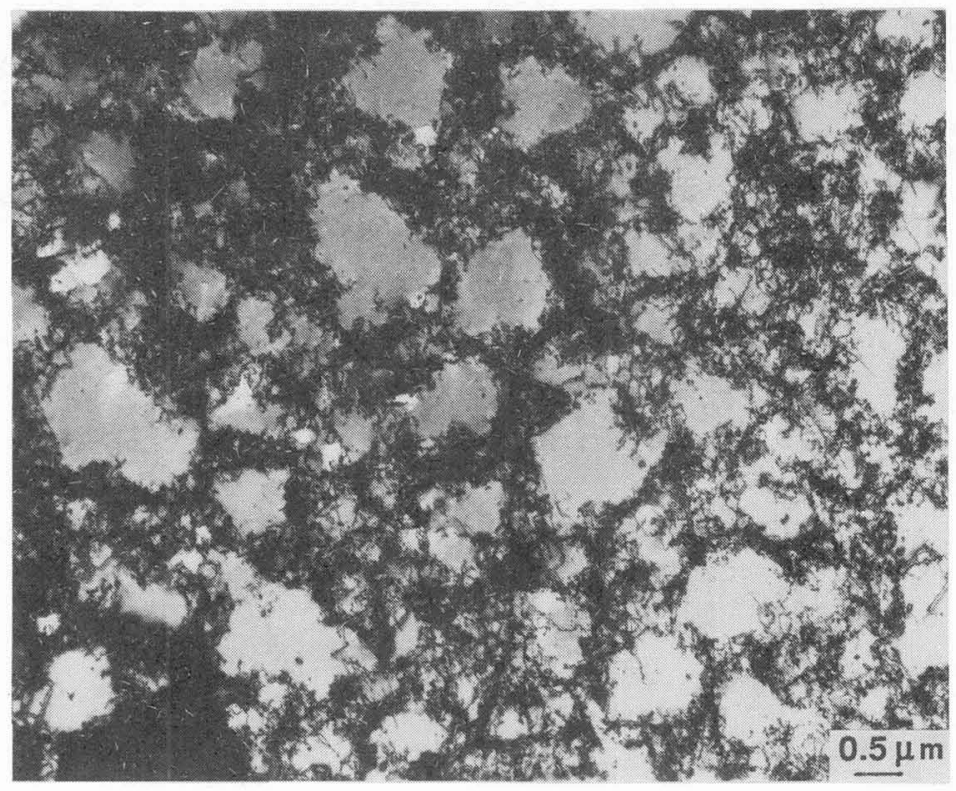

Fig. 5.- Well-defined dislocation cells formed in ramp-loading shock experiments. 


\section{4.- Summary}

Shock-recovery experiments to investigate the influence of loading path, at a constant peak shock pressure and pulse duration, from symmetric-shock loading (risetime $<1 \mathrm{~ns}$ ) to quasi-isentropic loading (risetime $\sim 1 \mu \mathrm{s}$ ) on the defect storage and post impact mechanical properties of copper have been conducted. Decreasing the strain-rate of loading is shown to increase both the defect storage and post-shock yield strength of copper. Independent of the loading rate the substructure evolution was observed to consist of dislocation cells and numerous dislocation loops. The effect of loading rate on post-shock substructure and mechanical response of impacted copper is postulated to be directly related to the amount of dislocation motion before interaction with other dislocations and to the amount of reversible dislocation motion and resultant annihilation during the rarefaction portion of the shock-release cycle.

\section{ACKNOWLEDGEMENTS}

Experimental contributions of J.N. Fritz, C.E. Frantz, C. Trujillo, M.F. Lopez, W. Wright, and W. Bast are gratefully acknowledged. This work was performed under the auspices of the U.S. Department of Energy.

\section{REFERENCES}

/1/ MURR, L.E., in Shock Waves and High Strain Rate Phenomena in Metals, Plenum Press, NY (1981) 607.

21 ZUKAS, E.G., Metals Eng. Quart.6(1966) 16.

B/ HUANG, S. and DING, F., in Shock Compression of Condensed Matter-1989, Elsevier Science Publishers, NY (1990) 313.

14/ Barker, L.M., in Shock Waves in Condensed Matter, Elsevier Science Publishers, NY (1984)

217.

15/ CHHABILDAS, L.C. and BARKER, L.M., in Shock Waves in Condensed Matter-1987,

Elsevier Science Publishers, NY (1988) 111.

16/ PEREZ, M., in Shock Compression of Condensed Matter-1989, Elsevier Science Publishers, NY (1990) 751.

nI $\quad$ GRAY III, G.T., FOLLANSBEE, P.S., and FRANTZ, C.E., Mat. Sci. Eng.A111 (1989) 9.

18/ GRAY III, G.T., in High Pressure Shock Compression of Solids, Springer-Verlag, West Germany (1991) in press.

19/ FOLLANSBEE, P.S. and GRAY III, G.T., Mat. Sci. Eng. (1991) in press.

/10/ GRAY III, G.T. and FOLLANSBEE, P.S., in Impact Loading and Dynamic Behavior of Materials, Deutsche Gesellschaft fuer Metallkunde, FRG (1988) 541. 